\title{
Clinical correlation among male infertility and overall male health: A systematic review of the literature
}

\author{
Francesco Del Giudice ${ }^{1,2, *}$ (1) , Alex M. Kasman ${ }^{2, *}$, Matteo Ferro ${ }^{3}$, Alessandro Sciarra $^{1}$ (10), Ettore De Berardinis ${ }^{1}$, \\ Federico Belladelli ${ }^{2,4}$, Andrea Salonia ${ }^{4}$ (D) Michael L. Eisenberg ${ }^{2,5}$ (i) \\ 'Department of Maternal-Infant and Urological Sciences, "Sapienza" Rome University, Policlinico Umberto I Hospital, Rome, Italy, ${ }^{2}$ Department of Urology, Stanford \\ University School of Medicine, Stanford, CA, USA, ${ }^{3}$ Division of Urology, European Institute of Oncology (IEO), Milan, ${ }^{4}$ Unit of Urology/Division of Oncology, IRCCS Ospedale \\ San Rafaele, URI, Milan, Italy, Department of Obstetrics and Gynecology, Stanford University School of Medicine, Stanford, CA, USA
}

Purpose: Ongoing evidence has suggested the role of male factor infertility as a potential predictor of mortality and general health status. The aim of the present review is to update the current knowledge base regarding the association between male factor infertility and general health through a critical review of the literature.

Materials and Methods: A systematic review of the literature was carried out from inception to November 2019 in order to evaluate significant associations between male infertility and adverse health outcomes such as cardiovascular, oncologic, metabolic and autoimmune diseases as well as overall mortality.

Results: In all, 27 studies met inclusion criteria and were critically examined. Five studies examined male infertility and cardiovascular disease risk, 11 examined oncologic risk (e.g., overall cancer risk, testis and prostate cancer), 8 examined aggregate chronic medical diseases and 5 infertility related to incidence of mortality, for a total of 599,807 men diagnosed with any male factor infertility covering a period from 1916 to 2016.

Conclusions: A man's fertility and overall health appear to be interconnected. Therefore, a diagnosis of male infertility may allow a window into future comorbidity and/or mortality which may help guide clinical decisions and counseling. Several possible etiologies such as genetic, epigenetic, developmental, and lifestyle-based factors need to be further evaluated in order to establish the underlying mechanisms between male infertility and health.

Keywords: Androgens; Health; Hypogonadism; Infertility, male; Mortality

This is an Open Access article distributed under the terms of the Creative Commons Attribution Non-Commercial License (http://creativecommons.org/licenses/by-nc/4.0) which permits unrestricted non-commercial use, distribution, and reproduction in any medium, provided the original work is properly cited.

\section{INTRODUCTION}

About $15 \%$ of couples do not achieve pregnancy within 1 year of attempting to conceive and thus are labeled as infertile [1,2]. Of those couples, male factor infertility is the underlying cause in $30 \%$ to $50 \%$ of cases [3]. Primary or secondary hypogonadism is a wellestablished predictor of male infertility as it can lead to alterations in all sperm parameters, with oligo-azoospermic men found to be hypogonadal in approximately $43 \%$ to $45 \%$ of cases which itself is associated

Received: 18 March, 2020 - Accepted: 12 April, 2020

Corresponding Author: Michael L. Eisenberg (iD https://orcid.org/0000-0001-5482-0141

Department of Urology, Stanford University School of Medicine, 300 Pasteur Drive, Stanford, CA 94305-5118, USA

TEL: +1-650-723-5700, FAX: +1-650-724-9609, E-mail: eisenberg@stanford.edu

*These authors contributed equally to this study and should be considered co-first authors. 
with impaired health (e.g., cardiovascular disease [CVD]) [4]. Recent literature has also identified lower sperm counts as an independent predictor of comorbidity and mortality [5-9].

As such male infertility has been proposed as an independent risk factor for poor health status and early mortality, while the etiology of this relationship remains unclear [10]. However, existing studies examining the prevalence of co-morbidities, morbidity, and mortality among infertile men are heterogeneous and contain often low level of evidence (LE). Given the increasing number of reports in this context, there is a need for synthesis of the data in order to better translate the conclusions into clinical practice and effective counseling.

As worldwide sperm counts continue to fall there may be an increase in the prevalence of male factor infertility $[9,10]$. As such, male infertility as a biomarker for future health and mortality will become more relevant. In the current study, we aim to systematically review the literature and present the findings regarding male infertility and comorbidities/mortality.

\section{MATERIALS AND METHODS}

\section{Evidence acquisition}

We performed a systematic review of the literature in PubMed, Embase, and Cochrane from inception to November 18th, 2019, without language restriction, to identify studies that examined male factor infertility and overall health, morbidity, and mortality. The reference lists of the included studies were also screened for relevant articles. Only original articles were included and critically evaluated. Case reports, abstracts and meeting reports were excluded from the analysis. Search terms included but were not limited to: male infertility, AND semen quality AND general health AND male comorbidities or male general dysfunction, AND male overall survival; secondary fields: male mortality; male hypogonadism; infertility and cardiovascular diseases; infertility and cancer development; infertility and chronic diseases; infertility and genetic associations; infertility and development associations. For all studies, we evaluated the LE according to the European Association of Urology (EAU) guidelines [11].

\section{Selection of the studies and criteria of inclusion}

Entry into the analysis was restricted to data collected from original studies and those that examined subfertile/ infertile males by semen analysis or those subjects with known male factor infertility. The reviewers utilized the Preferred Reporting Items for Systematic Reviews and Meta-Analyses (PRISMA) guidelines to develop the review
[12]. Three authors (FDG, FB, EDB, and AMK) independently screened the titles and abstracts of all articles using predefined inclusion criteria. The full-text articles were examined independently by four authors (MLE, FDG, MF, and $\mathrm{AS}$ ) to determine whether or not they met the inclusion criteria. Final inclusion was determined by consensus of all investigators. Selected articles meeting the inclusion criteria were then critically analyzed and data synthesized. The diagnosis of subfertile/infertility was based on failure to conceive for at least 12 months and/or on impaired semen analyses below the normal references values according to the World Health Organization (WHO) classification [13]) presence of other known male-related infertility factors (i.e., presence of varicocele; men seeking for fertility testing/ treatments).

The definition of CVD included a variety of different cardiovascular disorders, including ischemic coronary disease, cardiac failure and hypertension. All possible oncological associations with male infertility were examined. Analyses regarding chronic comorbidities and male infertility included metabolic syndrome and associated conditions such as obesity, insulin resistance, and dyslipidemia. All autoimmune disorder associations were included, such as multiple sclerosis (MS) and other rheumatological conditions (i.e., rheumatoid arthritis, psoriasis, Graves' disease, and autoimmune thyroiditis). Mortality including death from any causes retrieved.

\section{Assessment of quality for studies included}

The quality of the identified studies was assessed independently by two reviewers (FDG, FB) using the "Quality Assessment Tool for Observational Cohort and Cross-Sectional Studies," provided by the National Institute of Health (NIH) [14], by assessing the potential risk for selection bias, information bias, measurement bias, or confounding (confounding includes cointerventions, differences at baseline in patient characteristics, and other issues as shown in Supplementary Table 1) [15-41]. Studies were rated as good, fair, and poor quality, where high risk of bias translated to a rating of poor quality ("-") and low risk of bias translated to a rating of good quality (“+”).

\section{RESULTS}

\section{Search results}

The initial search yielded 334 articles (PubMed, 238; Cochrane, 62; and Embase, 34). One-hundred-ninety-six were excluded as they contained overlapping data or were duplicates appearing in multiple databases. Of the remaining 138 , 


\section{ICUROLOGY}

72 were further excluded since they did not examine male infertility (42), contained animal experiments (9), or were review papers or editorials (21). Full-text articles were then reevaluated and critically analyzed for the remaining 46 journal references. Within this in-depth review, a further 19 did not meet the inclusion criteria. The remaining 27 studies were included in our review (Fig. 1). No study was considered to be seriously flawed as per the "Quality Assessment Tool for Observational Cohort and Cross-Sectional Studies" [14]. Studies' risk to performance bias was moderately low across all the 27 studies. The risk of attrition bias due to incomplete outcome data was absent across all the studies (Supplementary Table 1) [15-41].

\section{Study locations and types}

Regarding infertility and CVD, 5 studies examined this association [15-19]. Four [15-17,19] were conducted in the United States (US), and 1 [18] in Europe (Italy). All of these were single-center retrospective surveys (Table 1). Eleven studies [20-30] examined infertility and its association with oncological malignancies. Of these, 7 [21,24-29] of these were conducted in the US, while 4 [20,22,23,30] were from Europe (Denmark and Sweden). Eight of eleven [23-30] were single-center retrospective population-based reviews while the remaining 3 [20-22] were case-control cohort studies (Table 1). For chronic disease association with male factor infertility, a total of 8 studies [16,18,31-36] were included. Of these, $3[16,33,36]$ were conducted in the US, 3 [18,32,34] in Italy, 1 [35] in Denmark, and 1 [31] in Qatar. Four [16,18,35,36] of 8 studies were single-center retrospective reviews while 3 [31-33] of them were single-center population-based cross-sectional studies and 1 [34] was a prospective case-control study (Table 1). Five [37-41] articles examined infertility and the risk of death. All 5 references were retrospective cohort studies. There was one [39] multicentered experience from the USA and the remaining four [37,38,40,41] from Europe (Denmark×2, Germany, Sweden) (Table 1). Of note, two studies (Eisenberg et al. [16] and Ferlin et al. [18]) analyzed multiple outcomes and therefore appear in multiple subsections.

\section{Study sample sizes, participant ages, and follow-up}

Given the lower LE, cross sectional and case-control studies included in the present review were separately considered with regard to available cumulative demographics characteristics. In total, nearly 600,000 men were included from 27 studies (Table 1) [15-41]. Within the 27 studies, there was a heterogenous population of men with regard to fertilitydiagnosis of infertility, semen parameters qualifying them as infertile, presenting for fertility evaluation, or were childless.

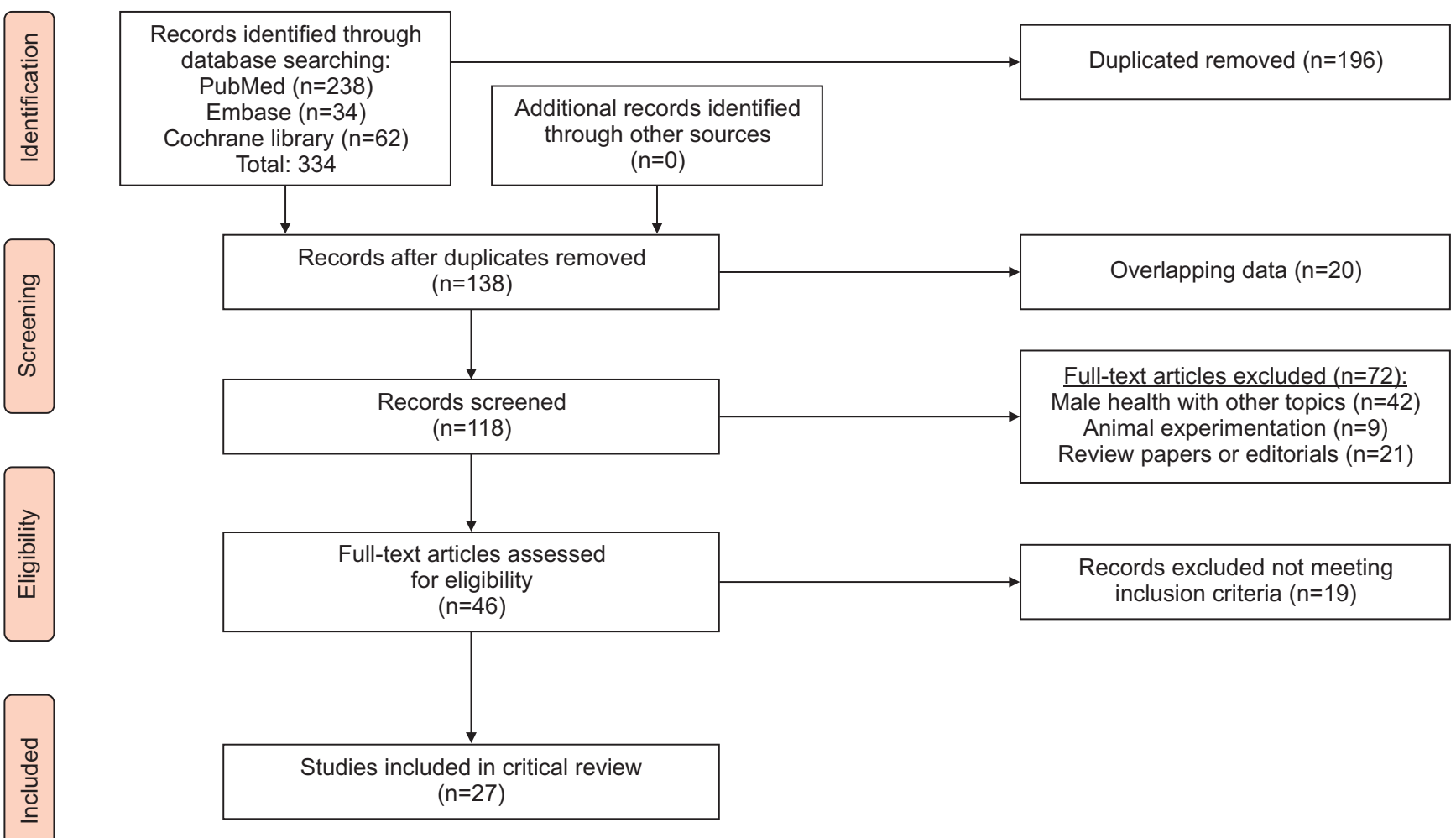

Fig. 1. Preferred Reporting Items for Systematic Reviews and Meta-Analyses (PRISMA) flow diagram. 


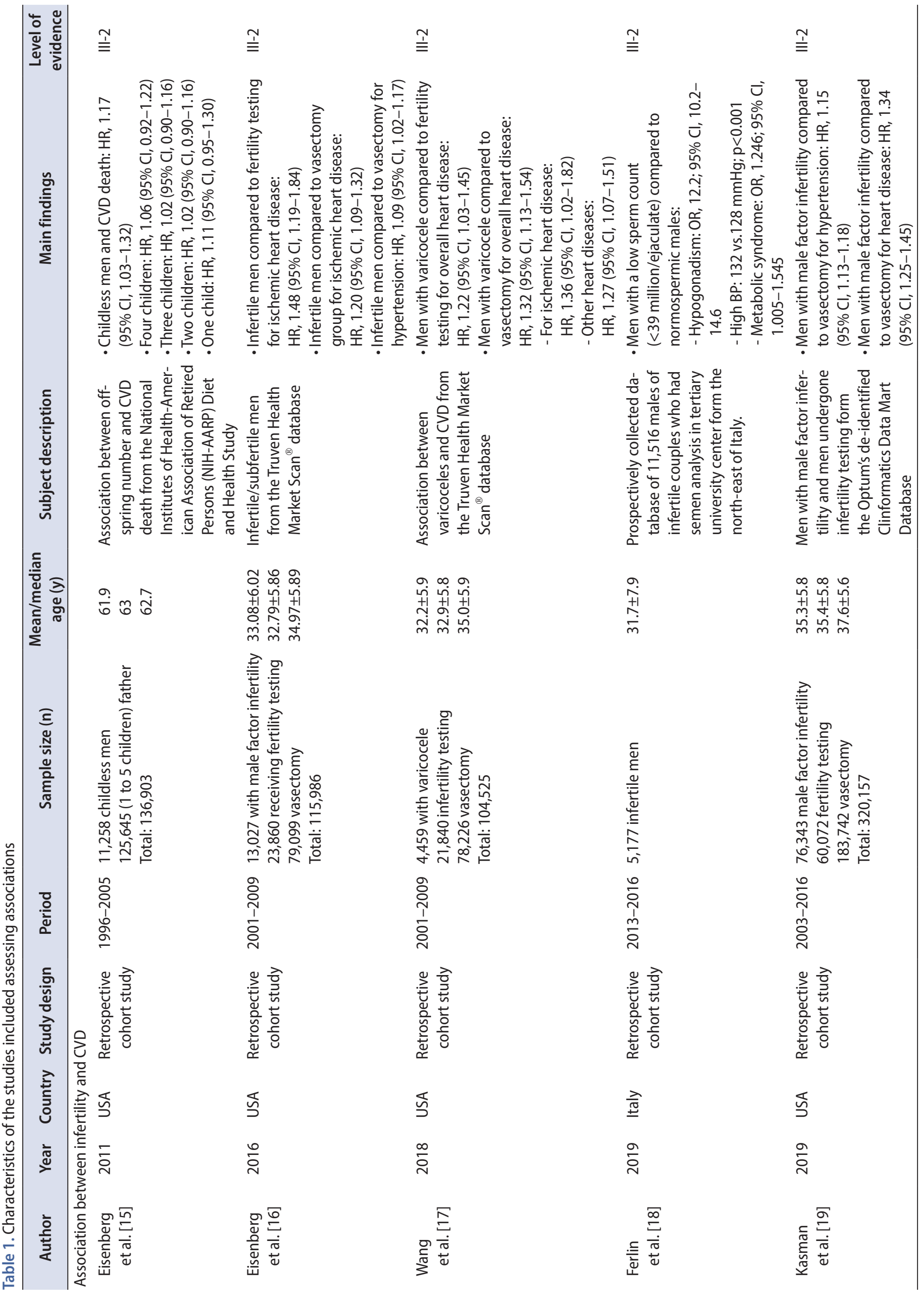




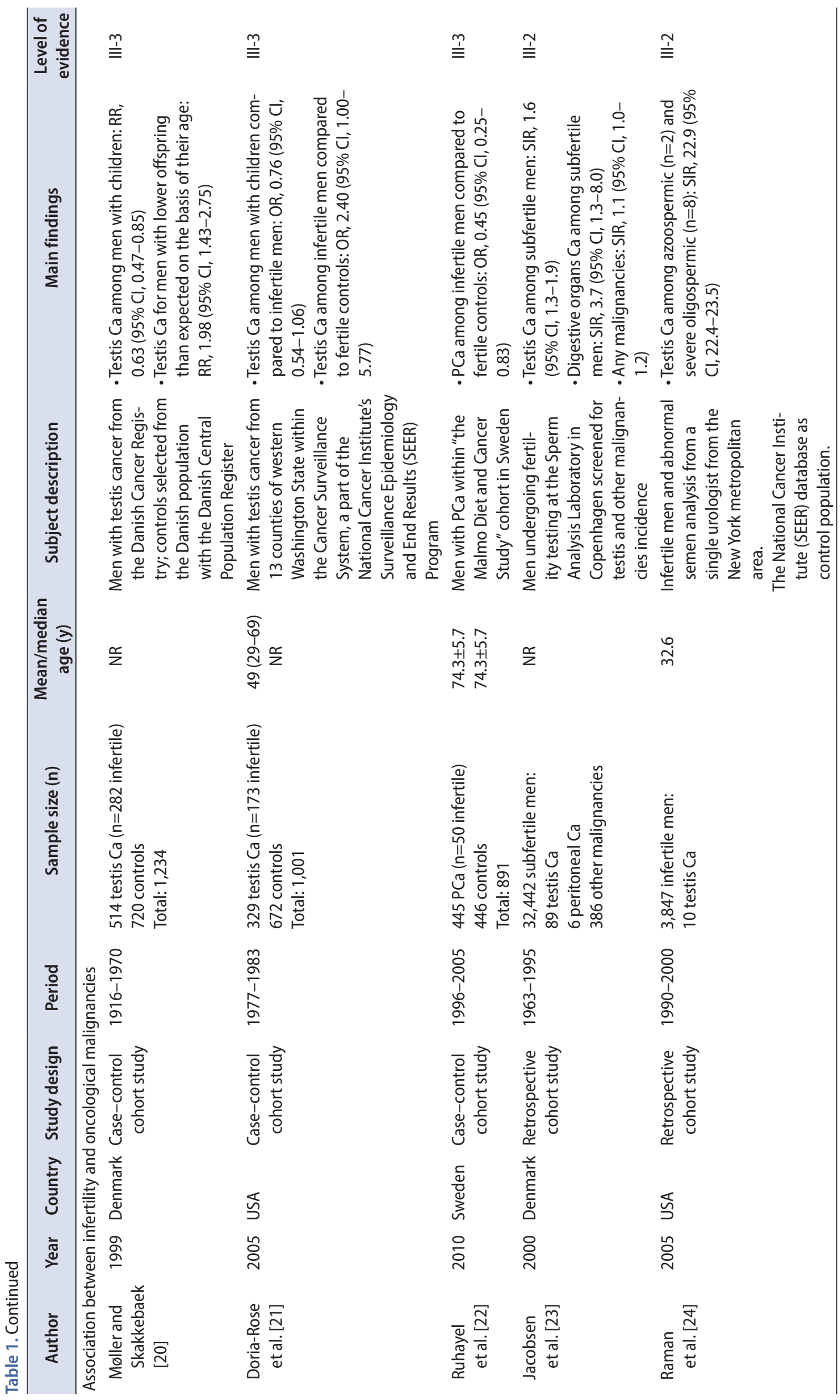




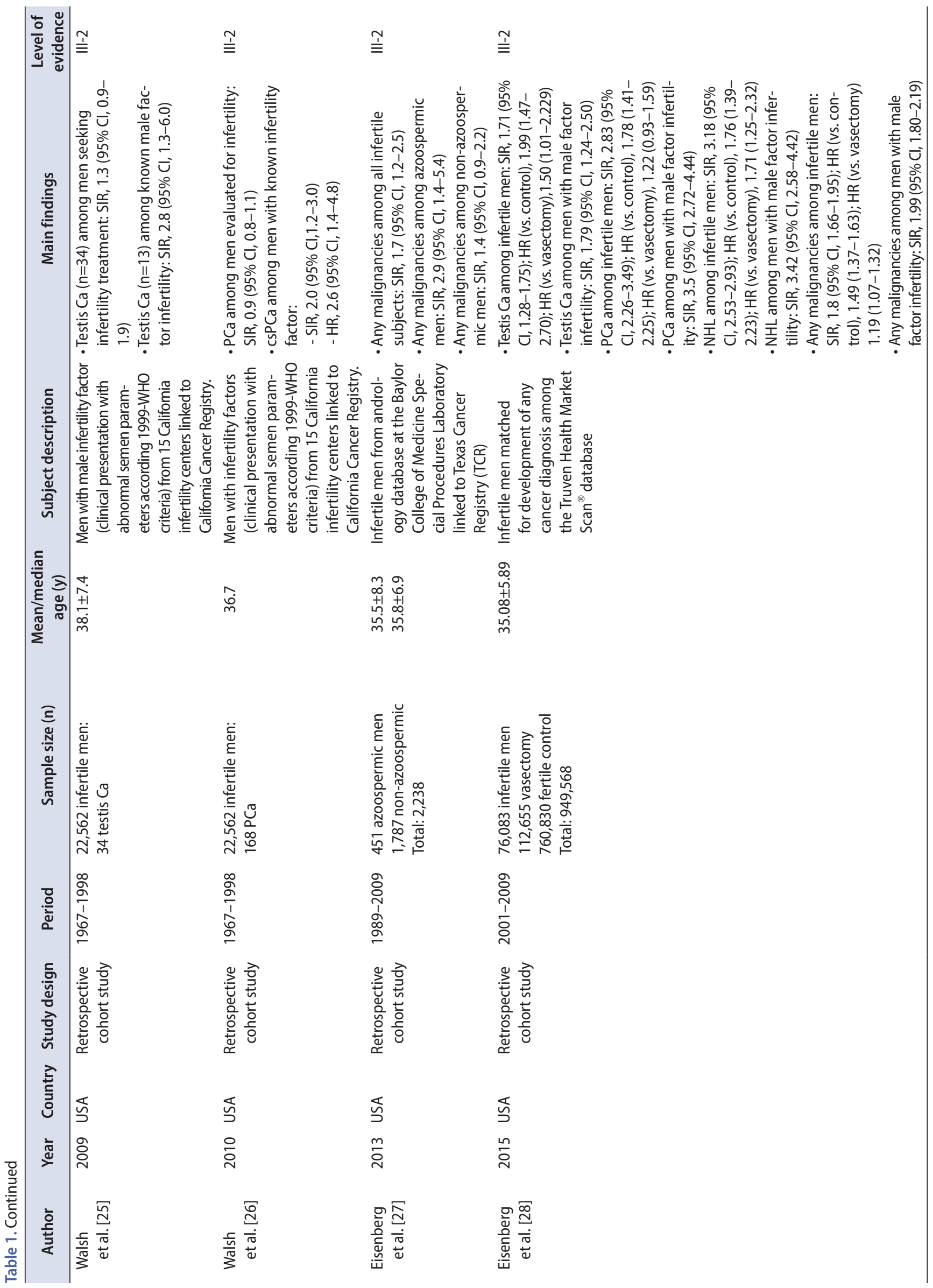




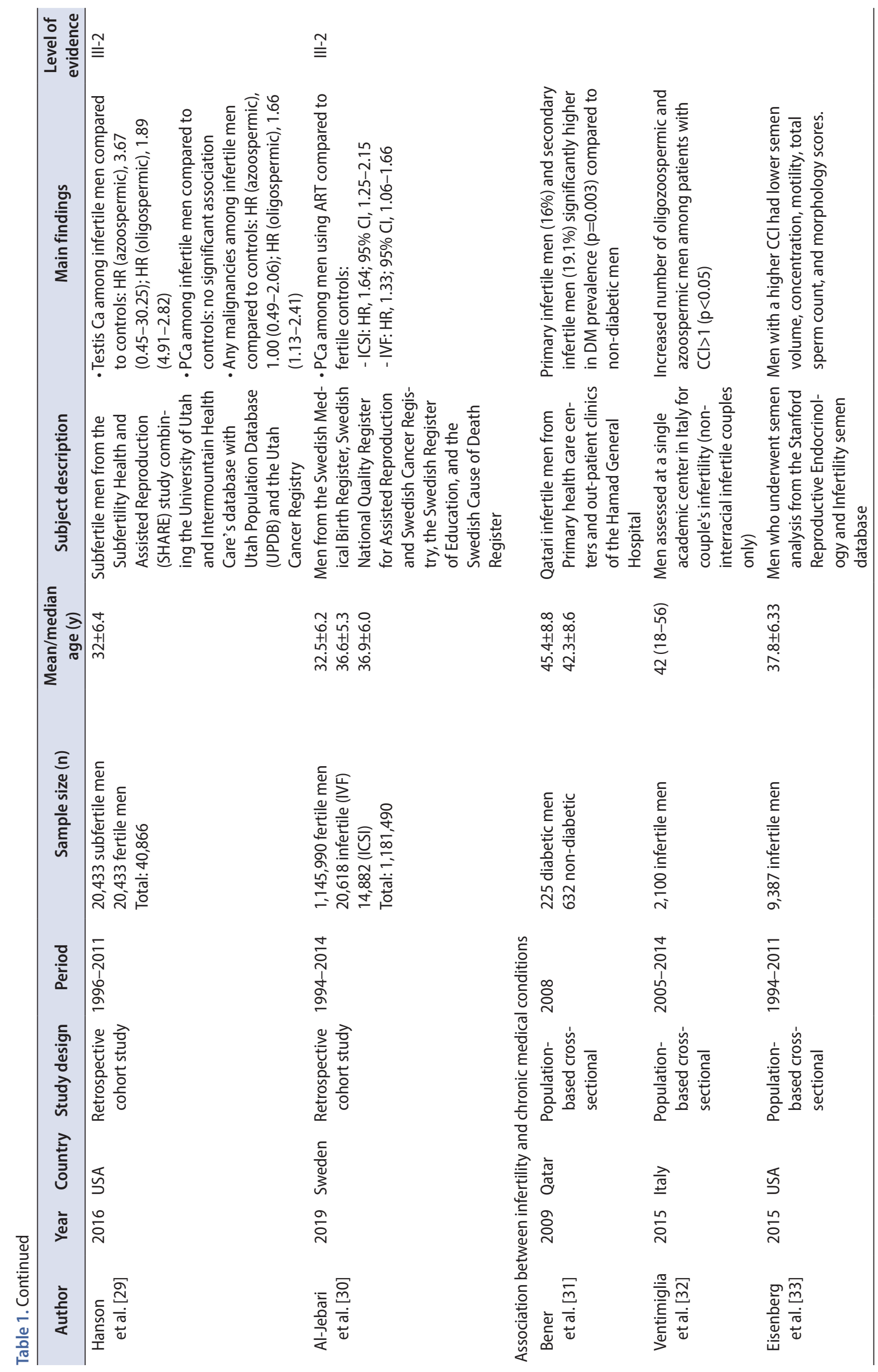




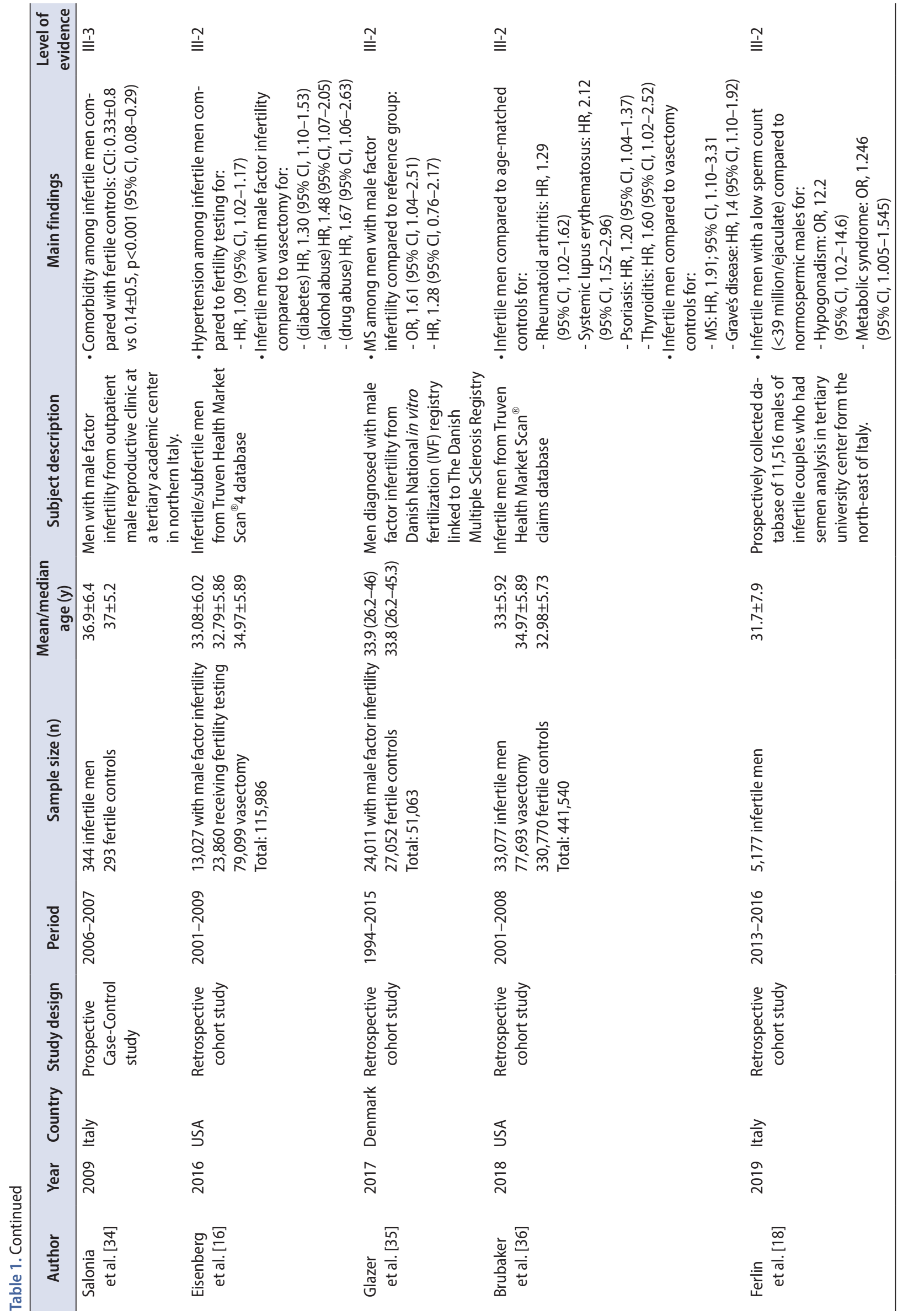




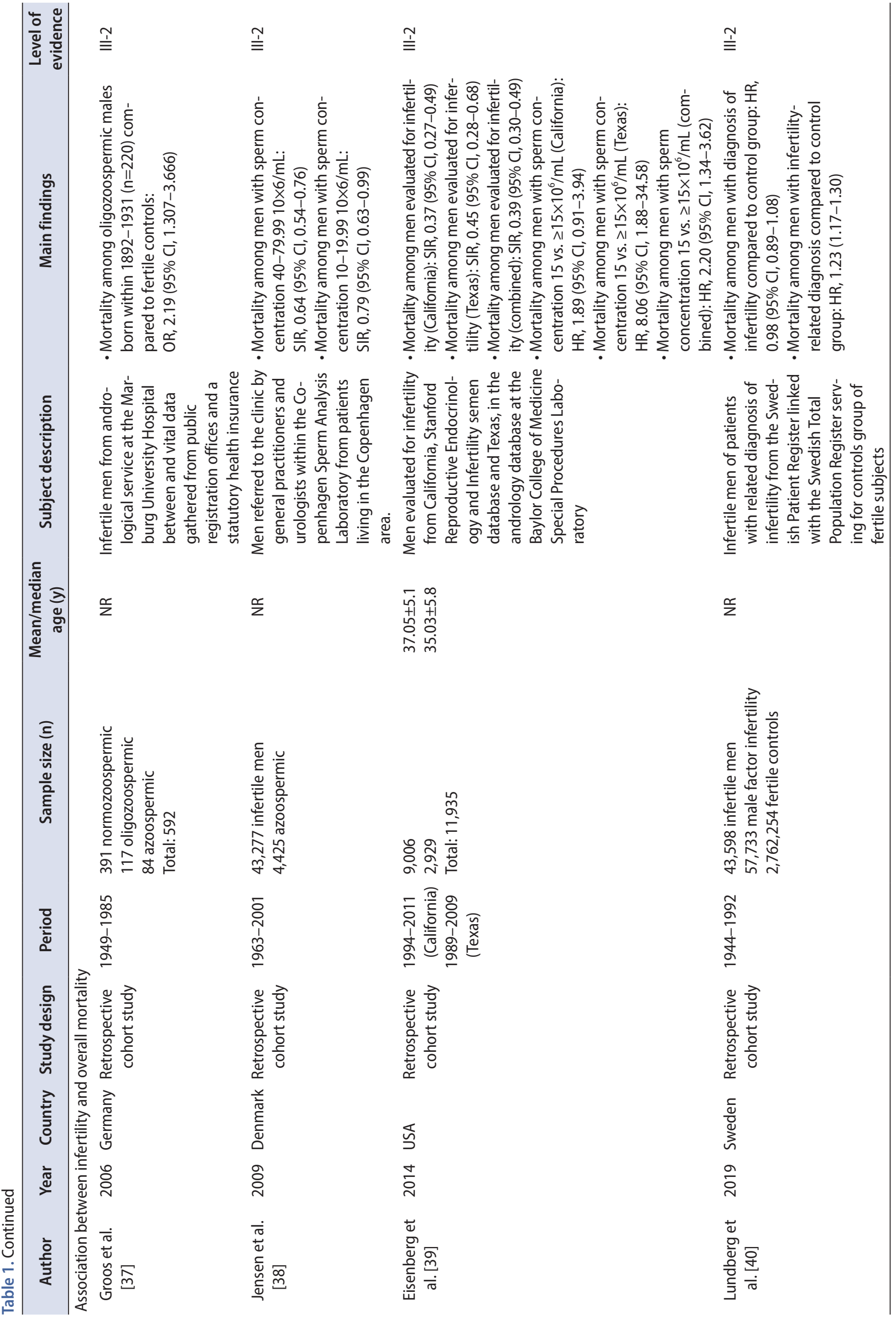




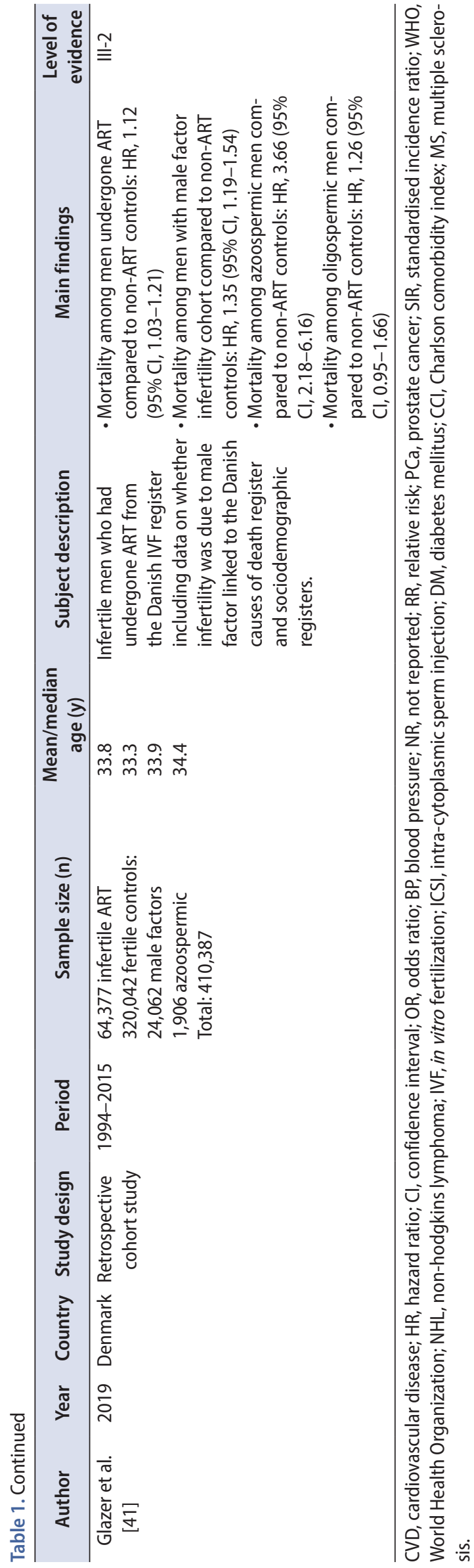

The sample sizes of each study varied from 857 to 9,387 men among case-control and cross-sectional analyses while the sample sizes for the retrospective cohort population studies ranged from 592 to 2,863,585 men. The mean age of infertile population across the studies varied from 37.8 to 74.3 years for cross sectional and case-control surveys versus 31.7 to 61.9 years for retrospective analyses.

\section{Male factor infertility and cardiovascular disorders}

Serum testosterone levels decline gradually with age in most men and several epidemiological/observational studies have demonstrated that low testosterone and male factor infertility are associated with an increased in CVD risk [4,42]. Moreover, previous meta-analysis studies have shown that even subclinical hypogonadism may affect the incidence of CVD and overall mortality related to CV events [43-45]. Therefore, authors have postulated that male infertility may be a marker for future cardiovascular risk (via hormonal pathways) or possibly exist as an independent risk factor.

A large retrospective study based on the National Institutes of Health-American Association of Retired Persons (NIH-AARP) Diet and Health registry on 136,903 men highlighted how compared with fathers, childless men had a $17 \%$ (hazard ratio [HR], 1.17; 95\% confidence interval [CI, 1.03-1.32) increased risk of death from CVD, and this elevated risk appeared to also extend to men with only one child [15]. However, the study lacked semen data and pregnancy intention of the fathers and therefore we cannot say that this association was due to fertility status or other confounding factors.

However, a large retrospective series of more than 13,000 men using the IBM MarketScan database demonstrated that male infertility was an independent predictor of increased risk of chronic medical conditions and, in particular, CVD [16]. Compared to the control group, infertile men had an increased incidence of hypertension, peripheral vascular disease and heart disease (HR, 1.09; 95\% CI, 1.02-1.17; HR, 1.52; 95\% CI, 1.12-2.07 and HR, 1.20; 95\% CI, 1.09-1.32, respectively). Furthermore, using the IBM MarketScan database, Wang et al. [17] demonstrated an association between the presence of varicocele and vascular disorders in a large retrospective cohort of 4,459 men. This is notable as varicocele represents a risk factor for infertility and occurs in about $15 \%$ of healthy men and is associated with primary infertility in up to $35 \%$ of men presenting for fertility evaluation. In this study, the authors found a higher incidence of heart disease in men with varicoceles compared to men who underwent infertility testing alone (HR, 1.22; 95\% CI, 1.03-1.45), and men who underwent vasectomy who served as a fertile control group 
(HR, 1.32; 95\% CI, 1.13-1.54). Interestingly, a sub-analysis of these patients stratified for symptomatic vs. asymptomatic varicoceles showed that only symptomatic varicoceles were associated with later health.

Two other studies demonstrated an association between infertility and CVD. In a study of 5,177 subjects, Ferlin et al. [18] showed that men with low sperm count ( $<39$ million/ ejaculate) were at a significantly higher risk of hypogonadism (odds ratio [OR], 12.2; 95\% CI, 10.2-14.6) and were overall at higher risk for chronic metabolic and cardiovascular disorders. Moreover, the authors concluded that low sperm count, independent of low serum $\mathrm{T}$, was associated with poorer metabolic, cardiovascular, and bone health status. However, the clinical significance of the differences was uncertain. For example, the differences in systolic blood pressure (128 vs. $132 \mathrm{mmHg}$ ), homeostatic model assessment (HOMA) index (1.8 vs. 1.9), and hemoglobin A1c (4.6\% vs. $4.4 \%$ ) between men with low and normal total sperm count were only modestly different. Finally, Kasman et al. [19] examined 136,416 males with infertility from the Optum Clinformatics Data Mart Database and found that male factor infertility was associated with the risk for cardiometabolic disease when compared to controls (vasectomized men) regardless of socioeconomic status, race, or geographic region. Men with male factor infertility had a higher risk of developing hypertension (HR, 1.15; 95\% CI, 1.13-1.18), and heart disease (HR, 1.34; 95\% CI, 1.25-1.45) compared to fertile controls.

\section{Male infertility and cancer risk}

While the treatment for many cancers are known to have a negative impact on male fertility, male infertility may also be associated with the future risk of cancer [46,47]. The underlying mechanism behind this potential link is unknown however genetic alterations may play a role. For example, one potential cause for male infertility is represented by disruptions in $M L H 1$ genes, and mutations in these genes can also lead to Lynch syndrome. Using ERCC1 (excision repair cross-complementing gene 1) or MSH2 (MutS homolog 2) knockouts, animal models have demonstrated that changes in these genes can lead to azoospermia in mice as well as increased early incidence of all malignant carcinomas [48-50]. However, the underlying etiology behind future cancer risk in infertile men remains unclear.

Among the 11 articles identified evaluating cancer risk in infertile men, the primary intent of the investigators was generally focused on establishing incidence of GU cancers (testicular, prostate cancer [PCa]) among infertile/subfertile subjects. One study which examined the impact of infertility and the overall risk of all cancers was the study of Eisen- berg et al. (2013) [27], In the retrospective cohort study from the Texas Cancer Registry, infertile men were found to be at higher risk of overall cancer (standardized incidence ratio [SIR], 1.7; 95\% CI, 1.2-2.5). Of relevance, azoospermic men had the highest risk of cancer (SIR, 2.9; 95\% CI, 1.4-5.4). A similar trend for overall cancer risk was confirmed in a 2015 analysis within the IBM MarketScan database which showed an overall HR of 1.49 (95\% CI, 1.37-1.63) compared to national US. estimate cancer incidence [28]. Moreover, this study confirmed previously observed relationships (i.e., male infertility with testis and PCa: HR, 1.99; 95\% CI, 1.47-2.70 and 178; 95\% CI, 1.41-2.25, respectively) and identified a higher risk of nonHodgkin lymphoma (HR, 176; 95\% CI, 139-2.23). While these data suggest that infertile men are at an increased risk of all cancers in the years after infertility evaluation, granular details about the men and their evaluation was not available to help elucidate the etiology of the association.

The association between semen quality and cancer remains uncertain due to heterogeneity in the literature. Hanson et al. [29] found that only oligozoospermia was associated with increased risk of all types of cancers, by an $\mathrm{HR}_{\text {Count }}$ 1.8 (95\% CI, 1.2-2.6), while those men with azoospermia did not have a significantly higher risk of cancer development (HR, 1.0; 95\% CI, 0.5-2.1). Finally, Jacobsen et al. [23] found that men with abnormal semen characteristics had a small increase (SIR, 1.1; 95\% CI, 1.0-1.2) in the incidence of any type of cancer (36 cases per 32,442 men) using linkage with the Danish Cancer Registry.

While some studies examined overall cancer risk, there has been relatively more focus on the future development of genitourinary malignancies in men diagnosed with infertility. Testicular cancer in relation to infertility has been well studied ( $\mathrm{n}=7$ studies [20,21,23-25,28,29]) while PCa has had less focus ( $n=5$ studies [22,26,28-30]). Overall, the literature demonstrated a significant increase in the risk of developing testis cancers if a man was diagnosed with infertility or has low semen parameters. The analysis of Raman et al. [24] revealed a 22-fold increased risk (SIR, 22.9; 95\% CI, 22.4-23.5) after examining 3,847 men evaluated from a single urologist in the New York metropolitan area during a 10-year period (1990 to 2000), and using the National Cancer Institute (NCI)Surveillance, Epidemiology, and End Results Program to identify a control population. Of note, Raman's cohort were a highly selected group of subjects with significant alterations (i.e, low sperm concentrations $\left[<20 \times 10^{6} / \mathrm{mL}\right]$ and concomitant defects in motility $[<50 \%]$ or morphology $[<50 \%]$ in semen parameters, leading to a diagnosis of infertility with the timing of cancer diagnosis uncertain. In contrast, other studies of male infertility have showed that infertile men may 
have a 2- to 3 -fold higher risk of testis cancer. For example, Walsh et al. [25] examined 4,459 men diagnosed with male factor infertility (i.e., clinical presentation with abnormal semen parameter-1999 WHO criteria) compared to 14,557 men with normal semen quality and reported a threefold higher risk of testis cancer (SIR, 2.8; 95\% CI, 13-6.0). Hanson et al. [29] also examined the association between male infertility and testis cancer. In this study, the authors demonstrated that infertile men had a higher incidence of testis cancer when compared to fertile controls. Of note, after stratifying within the infertile group, the investigators found that oligospermic subjects were at higher risk as compared with men with normal semen quality ( $\mathrm{HR}_{\text {Count, }} 10.3 ; 95 \% \mathrm{CI}, 4.1-26.2$ vs. HR, 2.9; 95\% CI, 1.2-6.7).

With regard of infertility and risk of $\mathrm{PCa}$, our review revealed several publications with conflicting results. In the Swedish study, Al-Jebari et al. [30] examined the risk of developing PCa among infertile men, retrieved from the Swedish Medical Birth Register and the Swedish Multi-generation Register who had achieved fatherhood through assisted reproductive technologies (ART). When the authors examined 35,500 men having undergone in vitro fertilization (IVF) or intracytoplasmic sperm injection (ICSI), compared to those who fathered children via natural conception, men having undergone ART had a significantly increased risk of development of PCa (HR, 164; 95\% CI, 125-215, for ICSI and HR, 133; 1.06-1.66, for IVF; respectively). Similar to the Swedish study, Eisenberg et al. [28] found an increased risk of PCa development (HR, 178; 95\% CI, 141-2.25) in men diagnosed with infertility when they examined the IBM MarketScan database from 2001 to 2009 which contains 76,083 number of men with infertility. However, the significance varied based on the control group examined. While the risk of PCa was higher with infertile men compared to an age-matched control, the risk was not significantly different compared to vasectomy men (i.e., arbitrarily considered fertile by definition). In contrast to other studies, Ruhayel et al. [22] utilized a nested case control design within the Malmo Diet and Health Study and observed that infertility status was associated with a lower risk of $\mathrm{PCa}(\mathrm{OR}$, 0.45; 95\% CI, 0.25-0.83). However, the study design may bias case ascertainment to men with less severe forms of PCa. Next, Hanson et al. [29] found no association with risk of development of PCa using a US cohort. With all studies, it should note that the majority of the men evaluated had not reached the average age of PCa diagnosis (66 years old in the US according to NCI), which may affect the correlation. Walsh et al. [26] revealed that men with male factor infertility had an increased risk of subsequent development of high grade $\mathrm{PCa}$ (SIR, 2.0; 95\% CI, 1.2-3.0; HR, 26; 95\% CI; 14-4.8) but not overall PCa (SIR,
0.9; 95\% CI, 0.8-1.1), thus suggesting that biology rather than screening bias may explain the etiology. Overall, these findings suggest that infertility status per se may be a risk factor for the development of PCa; however due to the heterogeneity in the literature, further studies are necessary.

\section{Male infertility and chronic medical conditions}

Factors such as smoking, increased body mass index (BMI), alcohol and/or drugs abuse, psychological stress have been associated with increased incidence of chronic diseases such as metabolic syndrome, erectile dysfunction, obesity, hematologic disorders, chronic kidney failure, liver disfunctions and in general with impaired HOMA indices [51-53]. Moreover, these identical factors have been implicated in the development of male infertility and decreased semen parameters [54-56]. However, as up to $10 \%$ of the genome is involved in male reproduction and there are only 25,000 genes, it is reasonable to postulate that genes involved in reproduction may also be expressed in other cell types [46]. Thus, defects in male reproduction may also signal an increased risk of the development of chronic disease (i.e, act as a biomarker).

Two large retrospective cohort studies focused on the relationship between male infertility and the risk of incident endocrine-metabolic syndromes, such as diabetes and metabolic syndrome. Wang et al. [17] utilized the IBM MarketScan database to examine more than 13,000 infertile men and found a significant association between the presence of a male factor infertility and the development of diabetes mellitus type 2, alcohol abuse, and drug abuse (HR, 1.30; 95\% CI, 1.10-1.53; HR, 148; 95\% CI, 1.07-2.05; HR, 1.67; 95\% CI, 1.06-2.63; respectively) compared to men who had only undergone fertility testing. A second analysis focused on the prevalence of infertility diagnosis from Italy was performed by Ferlin et al. [18]. The authors examined semen quality and reproductive function as a marker of general male health in infertile subjects who had semen analysis in tertiary university center in Italy from a prospectively collected database of 11,516 males. The authors found that men with lower sperm counts were at a higher risk of hypogonadism (OR, 12.2; 95\% CI, 10.2-14.6) and a variety constellation of conditions commonly associated with impaired general health, such as higher BMI, waist circumference, systolic pressure, low-density lipoprotein cholesterol, triglycerides, HOMA index and finally lower high-density lipoprotein cholesterol thus leading to a higher prevalence of metabolic syndrome (OR, 1.246; 95\% CI, 1.005-1.545) which was independent of their hypogonadism status. Overall, these two studies suggest that male factor infertility may be an independent predictor of future health.

We identified one prospective case-control study and 
two cross-sectional studies that examined the association between comorbidities, identified via the Charlson comorbidity index (CCI), and semen/hormonal parameters. Salonia et al. [34] evaluated 344 consecutive European Caucasian men with male factor infertility and demonstrated a higher prevalence of comorbidities as compared with fertile controls (CCI: 0.33 [0.8] vs. 0.14 [0.5], p<0.001; 95\% CI: 0.08-0.29). While $88.4 \%$ of the fertile controls had a $\mathrm{CCI}=0$, only $77.3 \%$ of the infertile men did $(\mathrm{p}<0.001)$. Moreover, at multivariable linear regression model, age, BMI and fertility status were all three found to independently predict CCI scores ( $\beta$ : 0.196, 0.161 and -0.199 respectively; $\mathrm{p}<0.001)$. This suggests that infertile patients have more comorbidities (e.g., cardiovascular disorders, pulmonary diseases, connective tissue disorders, liver diseases, DM and different malignant neoplasms) than fertile men. Similarly, the studies of Ventimiglia et al. [32] and Eisenberg et al. [33] confirmed an association among male factor infertility and increased prevalence chronic medical disorders. Different from the article from Salonia et al. [34], where the classic clinical WHO definition of infertility (i.e., $>12$-month failure with unprotected intercourses) was assumed, in these two studies patients were enrolled according to semen quality alterations. When viewed together, these studies concluded that male infertility or impaired semen parameters is associated with prevalent poor health.

As female factor infertility (e.g., endometriosis) has been associated with incident autoimmune disorders, investigators have also examined autoimmune dysfunction in male infertility patients [56]. While the etiology remains unknown, scientists have argued there may be an immune mediated mechanism to some forms of infertility [57,58]. A Danish group reviewed data from 24,011 infertile men from the Danish National IVF Registry and showed an increased prevalence (OR, 1.61; 95\% CI, 1.04-2.51) and incidence (HR, 1.28; 95\% CI, 0.76-2.17) of MS within men with known male factor infertility [35]. Brubaker et al. [36] examined IBM Market Scan claims database from 2001 to 2008 with 33,077 infertile men and found an association between autoimmune disorders, such as systemic lupus erythematosus, psoriasis, thyroiditis, MS and Grave's disease, and prior diagnosis of male infertility.

\section{Male factory infertility and mortality}

Finally, five studies [37-41] have suggested that male infertility is associated with mortality. Initially, a German cohort of 601 men over the span of 35 years who provided a semen sample as part of an andrological evaluation were found to have a higher rate of mortality if they were born between 1892 and 1931 [37]. For men born in other years, no association was identified. While the authors failed to establish a clear relationship between semen quality and mortality, the cohort included men raised in post World War II Germany. Thus, the results may not be generalizable.

More recently, other studies have explored contemporary cohorts to examine the association between male infertility and mortality. Jensen et al. [38] evaluated large cohort of Danish men who had semen analyses performed as part of an infertility evaluation and observed that mortality decreased as sperm concentration increased up to a threshold of $40 \mathrm{million} / \mathrm{mL}$. Subsequently, a study from the US [39] observed that men with two or more semen abnormalities had more than two-fold increased risk (HR, 2.57; 95\% CI, 1.26-5.23) of death. A Swedish study from Lundberg et al. [40] examined more than 40,000 men with infertility or infertility-related diagnosis and found no significant association among fertility status and overall death risk (HR, 0.98; 95\% CI, 0.89-1.08). While overall there was no association between infertility and death, after stratifying for confounders, the authors noted a 4.58-fold higher risk of death in men with a diagnosis of infertility before the age of 30 years, largely explained by cancer diagnosed before infertility. Here the authors suggested that prevalent disease likely led to the association of male infertility and mortality. Finally, a cohort study from the Danish IVF register reported the results from 64,563 men who had undergone medically assisted reproduction (MAR) between 1994 and 2015 [41]. When looking at the mortality ratios between men who conceived with MAR (all men regardless infertility) vs. those age-matched controls who naturally achieved pregnancy, no significant increased risk was detected (HR, 1.07; 95\% CI, 0.98-1.15). Of note, when stratifying by type of male factor infertility, azoospermic males had the highest risk of death (HR, 3.32; 95\% CI, 2.02-5.40) while the same association was not proved for oligospermic patients (HR, 1.14; 95\% CI, 0.87-1.50) or for those categorized as with "other male factor infertility" (HR, 1.10; 95\% CI, 0.75-1.61). As with all registry data, there is limited granular information about the infertile men thus other ailments or non-measured confounders may influence the results. However, the association with infertility and the dose response (as it relates to severity of male infertility diagnosis or level of semen impairment) does suggest a biological explanation.

\section{DISCUSSION}

Our review of the existing literature suggests and association between male factor infertility and somatic health. The literature is consistent in findings that demonstrate 
higher risk of CVD. Similarly, infertile males appear to be at higher risk of chronic disease regardless of sociodemographic factors. However, the association with cancers varies based on the specific cancer examined and conflicting results exist. Nevertheless, the etiology and clinical implications of the association require further elucidation especially to be able in future to balance the relative influence of the different infertility-related diagnosis (such as idiopathic, immunologic, varicocele, obstructive, cryptorchidism etc.) on the specific comorbidity development.

Overall, the literature suggests that semen parameters and overall testicular function may represent markers of general health $[59,60]$. As infertile men are evaluated early in life, there is an opportunity for health assessment, counseling, and disease prevention. This latest issue is of critical importance as typically infertile men represent a population of young subjects in which an early finding of hypogonadism, metabolic derangements, and overall risk of mortality may allow for more adequate prevention, management, follow-up, treatment, and lifestyle modifications.

The overall quality of the studies included in the present analysis was good, including six cross-sectional/casecontrol studies and twenty-one retrospective cohort-based analyses. The LE achieved varied from IV to III-2, which is considered overall good among epidemiological etiology-based studies but in general low. Although this systematic review has several strengths including the rigorous/standardized literature search and the quality assessment performed by three expert researchers in this field, several limitations of our analysis have to be acknowledged. First, the surveys within this research field are mainly directed by two infertility research poles in the US ( $\mathrm{n}=14$ studies) and in Europe ( $n=12$ studies) and may not be generalizable to other parts of the world. Therefore, we have to consider that the majority of the outcomes synthetized might be influenced by only selected investigators thus impacting on the overall risk of bias of the studies included. Second, the high level of heterogeneity among the different study designs, the presence of multiple variables which influence fertility outcomes as well as the differences of inclusion criteria for male infertility among the articles makes comparisons between studies challenging.

\section{CONCLUSIONS}

Current literature suggests an association between male infertility and risk of chronic disease, comorbidity, CVD, and cancer development. However, the literature remains small, with heterogenous study populations, many of which are retrospective in nature. There is a lack of prospective trials and the studies with the highest LE (i.e., III-2) have an insufficient adjustment of confounders that may preclude them from stating a definitive conclusion about male infertility as precursor of these outcomes. The exact biological mechanisms leading to such conclusions remains uncertain, but likely involves some combination of developmental, hormonal, lifestyle and genetic factors. Future studies will likely provide insight into this important topic.

\section{CONFLICTS OF INTEREST}

The authors have nothing to disclose.

\section{AUTHORS' CONTRIBUTIONS}

Research conception and design: Francesco Del Giudice and Michael L. Eisenberg. Data acquisition: Francesco Del Giudice, Federico Belladelli, Matteo Ferro, and Alessandro Sciarra. Statistical analysis: not applicable. Data analysis and interpretation: Francesco Del Giudice, Ettore De Berardinis, and Michael L. Eisenberg. Drafting of the manuscript: Francesco Del Giudice, Alex M. Kasman, and Michael L. Eisenberg. Critical revision of the manuscript: Andrea Salonia and Michael L. Eisenberg. Obtaining funding: not applicable. Administrative, technical, or material support: not applicable. Supervision: Michael L. Eisenberg, and Andrea Salonia. Approval of the final manuscript: all authors.

\section{SUPPLEMENTARY MATERIAL}

Scan this QR code to see the supplementary material, or visit https://www.icurology.org/src/sm/icurology-61-355-s001.pdf.

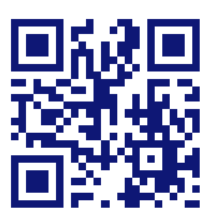

\section{REFERENCES}

1. Thoma ME, McLain AC, Louis JF, King RB, Trumble AC, Sundaram R, et al. Prevalence of infertility in the United States as estimated by the current duration approach and a traditional constructed approach. Fertil Steril 2013;99:1324-31.e1.

2. Louis JF, Thoma ME, Sørensen DN, McLain AC, King RB, Sundaram R, et al. The prevalence of couple infertility in the United States from a male perspective: evidence from a nationally representative sample. Andrology 2013;1:741-8. 
3. Brugh VM 3rd, Lipshultz LI. Male factor infertility: evaluation and management. Med Clin North Am 2004;88:367-85.

4. Ventimiglia E, Ippolito S, Capogrosso P, Pederzoli F, Cazzaniga W, Boeri L, et al. Primary, secondary and compensated hypogonadism: a novel risk stratification for infertile men. Andrology 2017;5:505-10.

5. Glazer CH, Bonde JP, Eisenberg ML, Giwercman A, Hærvig KK, Rimborg S, et al. Male infertility and risk of nonmalignant chronic diseases: a systematic review of the epidemiological evidence. Semin Reprod Med 2017;35:282-90.

6. Latif T, Kold Jensen T, Mehlsen J, Holmboe SA, Brinth L, Pors $\mathrm{K}$, et al. Semen quality as a predictor of subsequent morbidity: a Danish cohort study of 4,712 men with long-term follow-up. Am J Epidemiol 2017;186:910-7.

7. Latif T, Lindahl-Jacobsen R, Mehlsen J, Eisenberg ML, Holmboe SA, Pors K, et al. Semen quality associated with subsequent hospitalizations - can the effect be explained by socioeconomic status and lifestyle factors? Andrology 2018;6:42835.

8. Batty GD, Mortensen LH, Shipley MJ. Semen quality and risk factors for mortality. Epidemiology 2019;30:e19-21.

9. Levine H, Jørgensen N, Martino-Andrade A, Mendiola J, Weksler-Derri D, Mindlis I, et al. Temporal trends in sperm count: a systematic review and meta-regression analysis. Hum Reprod Update 2017;23:646-59.

10. Merzenich $\mathrm{H}$, Zeeb H, Blettner M. Decreasing sperm quality: a global problem? BMC Public Health 2010;10:24.

11. Aus G, Chapple C, Hanûs T, Irani J, Lobel B, Loch T, et al. The European Association of Urology (EAU) guidelines methodology: a critical evaluation. Eur Urol 2009;56:859-64.

12. Liberati A, Altman DG, Tetzlaff J, Mulrow C, Gøtzsche PC, Ioannidis JP, et al. The PRISMA statement for reporting systematic reviews and meta-analyses of studies that evaluate health care interventions: explanation and elaboration. J Clin Epidemiol 2009;62:e1-34.

13. World Health Organization. WHO laboratory manual for the examination and processing of human semen. 5th ed. Geneva: WHO Press; 2010.

14. National Heart, Lung, and Blood Institute (NHLBI). Quality assessment tool for observational cohort and cross-sectional studies [Internet]. Bethesda (MD): National Heart, Lung, and Blood Institute (NHLBI). Available from: https://www.nhlbi. nih.gov/health-topics/study-quality-assessment-tools.

15. Eisenberg ML, Park Y, Hollenbeck AR, Lipshultz LI, Schatzkin A, Pletcher MJ. Fatherhood and the risk of cardiovascular mortality in the NIH-AARP Diet and Health Study. Hum Reprod 2011;26:3479-85.

16. Eisenberg ML, Li S, Cullen MR, Baker LC. Increased risk of incident chronic medical conditions in infertile men: analysis of United States claims data. Fertil Steril 2016;105:629-36.

17. Wang NN, Dallas K, Li S, Baker L, Eisenberg ML. The association between varicocoeles and vascular disease: an analysis of U.S. claims data. Andrology 2018;6:99-103.

18. Ferlin A, Garolla A, Ghezzi M, Selice R, Palego P, Caretta N, et al. Sperm count and hypogonadism as markers of general male health. Eur Urol Focus 2019 Aug 16 [Epub]. https://doi.org/10. 1016/j.euf.2019.08.001.

19. Kasman AM, Li S, Luke B, Sutcliffe AG, Pacey AA, Eisenberg ML. Male infertility and future cardiometabolic health: does the association vary by sociodemographic factors? Urology 2019;133:121-8.

20. Møller H, Skakkebaek NE. Risk of testicular cancer in subfertile men: case-control study. BMJ 1999;318:559-62.

21. Doria-Rose VP, Biggs ML, Weiss NS. Subfertility and the risk of testicular germ cell tumors (United States). Cancer Causes Control 2005;16:651-6.

22. Ruhayel Y, Giwercman A, Ulmert D, Rylander L, Bjartell A, Manjer J, et al. Male infertility and prostate cancer risk: a nested case-control study. Cancer Causes Control 2010;21:1635-43.

23. Jacobsen R, Bostofte E, Engholm G, Hansen J, Olsen JH, Skakkebaek NE, et al. Risk of testicular cancer in men with abnormal semen characteristics: cohort study. BMJ 2000;321:789-92.

24. Raman JD, Nobert CF, Goldstein M. Increased incidence of testicular cancer in men presenting with infertility and abnormal semen analysis. J Urol 2005;174:1819-22; discussion 1822.

25. Walsh TJ, Croughan MS, Schembri M, Chan JM, Turek PJ. Increased risk of testicular germ cell cancer among infertile men. Arch Intern Med 2009;169:351-6.

26. Walsh TJ, Schembri M, Turek PJ, Chan JM, Carroll PR, Smith JF, et al. Increased risk of high-grade prostate cancer among infertile men. Cancer 2010;116:2140-7.

27. Eisenberg ML, Betts P, Herder D, Lamb DJ, Lipshultz LI. Increased risk of cancer among azoospermic men. Fertil Steril 2013;100:681-5.

28. Eisenberg ML, Li S, Brooks JD, Cullen MR, Baker LC. Increased risk of cancer in infertile men: analysis of U.S. claims data. J Urol 2015;193:1596-601.

29. Hanson HA, Anderson RE, Aston KI, Carrell DT, Smith KR, Hotaling JM. Subfertility increases risk of testicular cancer: evidence from population-based semen samples. Fertil Steril 2016;105:322-8.e1.

30. Al-Jebari Y, Elenkov A, Wirestrand E, Schütz I, Giwercman A, Lundberg Giwercman Y. Risk of prostate cancer for men fathering through assisted reproduction: nationwide population based register study. BMJ 2019;366:15214.

31. Bener A, Al-Ansari AA, Zirie M, Al-Hamaq AO. Is male fertility associated with type 2 diabetes mellitus? Int Urol Nephrol 2009;41:777-84. 
32. Ventimiglia E, Capogrosso P, Boeri L, Serino A, Colicchia M, Ippolito $\mathrm{S}$, et al. Infertility as a proxy of general male health: results of a cross-sectional survey. Fertil Steril 2015;104:48-55.

33. Eisenberg ML, Li S, Behr B, Pera RR, Cullen MR. Relationship between semen production and medical comorbidity. Fertil Steril 2015;103:66-71.

34. Salonia A, Matloob R, Gallina A, Abdollah F, Saccà A, Briganti A, et al. Are infertile men less healthy than fertile men? Results of a prospective case-control survey. Eur Urol 2009;56:1025-31.

35. Glazer CH, Tøttenborg SS, Giwercman A, Bräuner EV, Eisenberg ML, Vassard D, et al. Male factor infertility and risk of multiple sclerosis: a register-based cohort study. Mult Scler 2017:1352458517734069.

36. Brubaker WD, Li S, Baker LC, Eisenberg ML. Increased risk of autoimmune disorders in infertile men: analysis of US claims data. Andrology 2018;6:94-8.

37. Groos S, Krause W, Mueller UO. Men with subnormal sperm counts live shorter lives. Soc Biol 2006;53:46-60.

38. Jensen TK, Jacobsen R, Christensen K, Nielsen NC, Bostofte E. Good semen quality and life expectancy: a cohort study of 43,277 men. Am J Epidemiol 2009;170:559-65.

39. Eisenberg ML, Li S, Behr B, Cullen MR, Galusha D, Lamb DJ, et al. Semen quality, infertility and mortality in the USA. Hum Reprod 2014;29:1567-74.

40. Lundberg FE, Johansson AL, Ludvigsson JF. Mortality in 43,598 men with infertility - a Swedish nationwide populationbased cohort study. Clin Epidemiol 2019;11:645-57.

41. Glazer CH, Eisenberg ML, Tøttenborg SS, Giwercman A, Flachs EM, Bräuner EV, et al. Male factor infertility and risk of death: a nationwide record-linkage study. Hum Reprod 2019; 34:2266-73.

42. Kloner RA, Carson C 3rd, Dobs A, Kopecky S, Mohler ER 3rd. Testosterone and cardiovascular disease. J Am Coll Cardiol 2016;67:545-57.

43. Ponce OJ, Spencer-Bonilla G, Alvarez-Villalobos N, Serrano V, Singh-Ospina N, Rodriguez-Gutierrez R, et al. The efficacy and adverse events of testosterone replacement therapy in hypogonadal men: a systematic review and meta-analysis of randomized, placebo-controlled trials. J Clin Endocrinol Metab 2018 Mar 17 [Epub]. https://doi.org/10.1210/jc.2018-00404.

44. Corona G, Rastrelli G, Di Pasquale G, Sforza A, Mannucci E, Maggi M. Endogenous testosterone levels and cardiovascular risk: meta-analysis of observational studies. J Sex Med 2018;15: 1260-71.

45. Mangolim AS, Brito LAR, Nunes-Nogueira VS. Effectiveness of testosterone therapy in obese men with low testosterone levels, for losing weight, controlling obesity complications, and preventing cardiovascular events: protocol of a systematic review of randomized controlled trials. Medicine (Baltimore) 2018;97:e0482.

46. Matzuk MM, Lamb DJ. The biology of infertility: research advances and clinical challenges. Nat Med 2008;14:1197-213.

47. Feitsma H, Leal MC, Moens PB, Cuppen E, Schulz RW. Mlh1 deficiency in zebrafish results in male sterility and aneuploid as well as triploid progeny in females. Genetics 2007;175:15619.

48. Guerri G, Maniscalchi T, Barati S, Busetto GM, Del Giudice F, De Berardinis E, et al. Non-syndromic monogenic male infertility. Acta Biomed 2019;90(10-S):62-7.

49. Terribas E, Bonache S, García-Arévalo M, Sánchez J, Franco E, Bassas L, et al. Changes in the expression profile of the meiosis-involved mismatch repair genes in impaired human spermatogenesis. J Androl 2010;31:346-57.

50. Stupart D, Win AK, Winship IM, Jenkins M. Fertility after young-onset colorectal cancer: a study of subjects with Lynch syndrome. Colorectal Dis 2015;17:787-93.

51. Sermondade N, Faure C, Fezeu L, Shayeb AG, Bonde JP, Jensen TK, et al. BMI in relation to sperm count: an updated systematic review and collaborative meta-analysis. Hum Reprod Update 2013;19:221-31.

52. Busetto GM, Del Giudice F, Virmani A, Sciarra A, Maggi M, Ferro M, et al. Body mass index and age correlate with antioxidant supplementation effects on sperm quality: post hoc analyses from a double-blind placebo-controlled trial. Andrologia 2020;52:e13523.

53. Busetto GM, Agarwal A, Virmani A, Antonini G, Ragonesi G, Del Giudice F, et al. Effect of metabolic and antioxidant supplementation on sperm parameters in oligo-asthenoteratozoospermia, with and without varicocele: a double-blind placebo-controlled study. Andrologia 2018;50:e12927.

54. Guerri G, Maniscalchi T, Barati S, Dhuli K, Busetto GM, Del Giudice F, et al. Syndromic infertility. Acta Biomed 2019;90(10S):75-82.

55. Del Giudice F, Busetto GM, De Berardinis E, Sperduti I, Ferro M, Maggi M, et al. A systematic review and meta-analysis of clinical trials implementing aromatase inhibitors to treat male infertility. Asian J Androl 2019 Oct 15 [Epub]. https://doi.org/ 10.4103/aja.aja_101_19.

56. Giovannone R, Busetto GM, Antonini G, De Cobelli O, Ferro $\mathrm{M}$, Tricarico S, et al. Hyperhomocysteinemia as an early predictor of erectile dysfunction: International Index of Erectile Function (IIEF) and penile Doppler ultrasound correlation with plasma levels of homocysteine. Medicine (Baltimore) 2015;94:e1556.

57. Shigesi N, Kvaskoff M, Kirtley S, Feng Q, Fang H, Knight JC, et al. The association between endometriosis and autoimmune diseases: a systematic review and meta-analysis. Hum Reprod Update 2019;25:486-503. 
58. Ortona E, Pierdominici M, Maselli A, Veroni C, Aloisi F, Shoenfeld Y. Sex-based differences in autoimmune diseases. Ann Ist Super Sanita 2016;52:205-12.

59. Sicotte NL, Giesser BS, Tandon V, Klutch R, Steiner B, Drain AE, et al. Testosterone treatment in multiple sclerosis: a pilot study. Arch Neurol 2007;64:683-8.

60. Kasman AM, Del Giudice F, Eisenberg ML. New insights to guide patient care: the bidirectional relationship between male infertility and male health. Fertil Steril 2020;113:469-77. 